\title{
Clinicopathological Study of Patients Undergoing Resection of Hilar Cholangiocarcinoma
}

\author{
ATSUHIRO HIDAKA \\ Department of Surgery, Kurume University School of Medicine, \\ Kurume 830-0011, Japan
}

Received 20 September 2007, accepted 20 November 2007

\begin{abstract}
Summary: Of the patients who underwent resection of hilar cholangiocarcinoma, those who received palliative surgery, or could not be followed up clinicopathologically, were excluded from this study. In the remaining 37 patients, the cumulative postoperative survival rate (simply referred to as the cumulative survival rate below) was analyzed according to gross types, histopathological parameters, final stages, and final curability. These patients showed a 5-year survival rate of $17.7 \%$ and a $50 \%$ survival of 2.2 years. By gross type, patients with a localized papillary or nodular expansion type tended to have a better survival rate than those with an invasive papillary or nodular expansion type. A greater histological depth of invasion tended to be associated with a poorer prognosis: the $\mathrm{s}(-)$ group, that is, a group of patients without serosal exposure of cancer (invasion depths of $\mathrm{m}, \mathrm{fm}$, and ss) had a significantly better prognosis than the $\mathrm{s}(+)$ group, a group of patients with serosal exposure of cancer (invasion depths of se and si). Other histopathological parameters, such as ly, pn, pHinf, pHM, and $\mathrm{pEM}$, were associated with significant prognostic differences. By final stage, the stage I/II group and stage III or higher group showed a particularly significant difference in prognosis. By final curability, the curability A/B group had a significantly better prognosis than the curability $\mathrm{C}$ group. Taken together, surgery providing curability A and B promises a good long-term prognosis. Therefore, it is important that efforts are made to detect cancer early, adequately evaluate the degree of cancer extension, and determine the extent of resection and the surgical technique.
\end{abstract}

Key words hilar cholangiocarcinoma, cumulative survival rate, histopathological parameter, final stage, final curability

\section{INTRODUCTION}

Recent advances in diagnostic imaging techniques and perioperative management have been improving treatment results in cholangiocarcinomas. However, many hilar cholangiocarcinomas cannot be radically resected because of their state of extension. Even if they are resectable, their long-term prognosis is far from satisfactory. The accurate preoperative staging of cholangiocarcinomas and their reliable resection based on surgical anatomy are important. In this study, we clinicopathologically analyzed patients who had un- dergone resection of hilar cholangiocarcinoma in our department, and determined conditions for achieving long-term survival.

\section{MATERIALS AND METHODS}

Sixty-three patients had undergone resection of hilar cholangiocarcinoma in Kurume University Hospital between 1997 and 2003. In those, 37 patients were studied, excluding those who received palliative surgery (16 patients), or could not be followed up clinicopathologically (10 patients). They consisted of 20 men

Address for corresponding author: Atsuhiro Hidaka M.D., Department of Surgery, Kurume University School of Medicine, 67 Asahi-machi, Kurume 8300011, Japan. Tel: 0942-31-7567 Fax: 0942-35-8967 E-mail: atushiro@st-mary-med.or.jp 
TABLE 1.

Breakdown of surgical techniques

\begin{tabular}{lcccc}
\hline \multicolumn{1}{c}{ Surgical technique } & $\begin{array}{c}\text { Number of } \\
\text { patients }\end{array}$ & $\begin{array}{c}\text { Caudate } \\
\text { lobectomy }\end{array}$ & $\begin{array}{c}\text { Concomitant vascular } \\
\text { resection }\end{array}$ & Pancreaticoduodenectomy \\
\hline Left hepatic lobectomy & 8 & 6 & 1 & 0 \\
Extended left hepatic lobectomy & 6 & 2 & 4 & 0 \\
Right hepatic lobectomy & 4 & 2 & 0 & 1 \\
Extended right hepatic lobectomy & 9 & 5 & 0 & 0 \\
Right hepatic trisegmentectomy & 1 & 0 & 0 & 0 \\
S4a+S5 liver segmentectomy & 5 & 0 & 1 & 1 \\
Others & 4 & 0 & 7 & 2 \\
\hline \multicolumn{1}{c}{ Total } & 37 & 15 & & 4 \\
\hline
\end{tabular}

and 17 women, ranging in age from 52 to 77 years, with a mean of $63.7 \pm 6.47$ years. All patients had undergone hepatectomy with extrahepatic bile duct resection (Table 1). Cumulative postoperative survival rates were compared among gross types, clinicopathological parameters, final stages, and final curability. Positive and negative groups were defined by scores of 0 and 1 or higher, respectively, for ly, v, pn, pHinf, pGinf, and $\mathrm{pN}$. Negative and positive resection margins were defined by a score of 0 or 1 and that of 2 , respectively, for $\mathrm{pHM}, \mathrm{pDM}$, and $\mathrm{pEM}$. Because of the small number of combined resections, cumulative postoperative survival rates were not compared among pPanc, pDu, pPV, and pA. Pathological examination was performed according to the General Rules for Surgical and Pathological Studies on Cancer of the Biliary Tract by the Japanese Society of Biliary Surgery [1]. Survival curves by parameters were calculated by the Kaplan-Meier method. The significance of differences was analyzed by the log rank test. Multivariate analysis was performed by using cluster analysis. $P$ values less than 0.05 were considered significant.

\section{RESULTS}

The patients had overall 3- and 5-year survival rates of $28.2 \%$ and $17.7 \%$, respectively (Fig. 1), and a $50 \%$ survival time of 2.2 years.

\section{Survival rates by gross type}

Cancers were classified into localized and invasive papillary or nodular expansion types. The 3- and 5 -year survival rates of patients with localized types were both $33.3 \%$, whereas the 3- and 5-year survival rates of those with invasive types were $27.4 \%$ and $15.6 \%$, respectively. The invasive types tended to lead

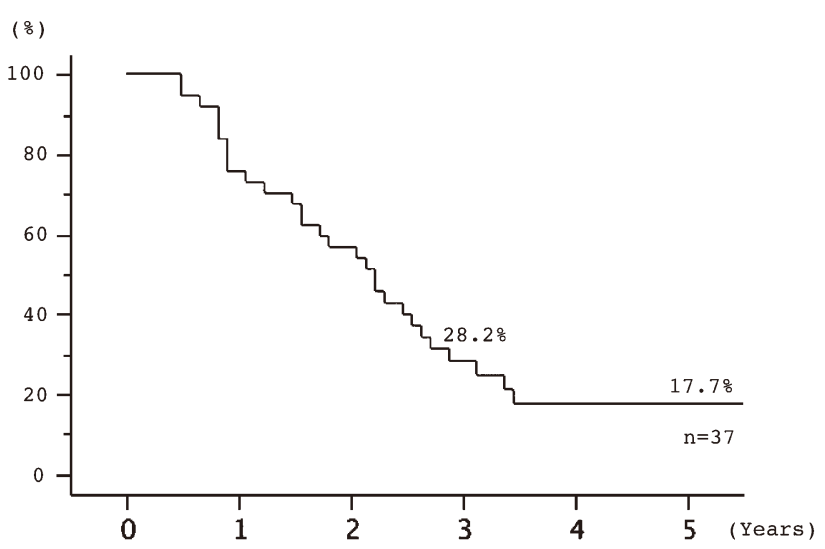

Fig. 1. Survival rates in resected patients with hilar cholangiocarcinoma.

to better survival rates than their localized counterparts, with no significant differences.

\section{Survival rates by histological type}

Thirty-six cancers were analyzed, excluding 1 of unknown histopathology (no histopathological record). Cancers were divided into well-differentiated papillary (pap) and tubular adenocarcinomas (tubl) and other histological types. The 3- and 5-year survival rates in the well-differentiated types were $37.9 \%$ and $25.2 \%$, respectively, and the 3 -year survival rate in other histological types was $18.8 \%$. The well-differentiated types tended to lead to a better survival rate, with no significant difference from that in the other types.

Survival rates by histological depth of cancer invasion

A greater depth of invasion tended to be associated with a poorer prognosis. The 3- and 5-year survival rates were $55.0 \%$ and $45.8 \%$, respectively, in the s(-) 
group, that is, a group without serosal exposure of cancers with invasion depths of $\mathrm{m}$, fm, and ss, and $11.6 \%$ and $0 \%$, respectively, in the $s(+)$ group with serosal exposure of cancers with invasion depths of se and si. Thus, the prognosis was significantly better in the $s(-)$ group (Fig. 2).

Survival rates by the presence or absence of neurovascular invasion

Lymphatic invasion (ly): Thirty-six cancers were analyzed, excluding 1 of unknown histopathology. The

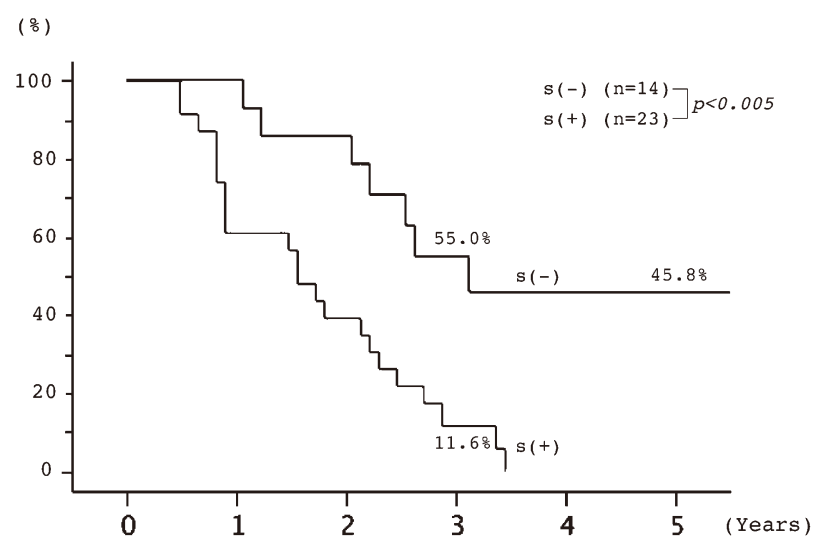

Fig. 2. Survival rates by histological depth of invasion.
3- and 5-year survival rates in the $1 \mathrm{y}(-)$ group were significantly higher, both at $68.6 \%$, than $20.1 \%$ and $8.1 \%$, respectively, in the ly $(+)$ group.

Venous invasion (v): Twenty-nine cancers were analyzed, excluding 8 of unknown histopathology. The 3 - and 5-year survival rates in the $\mathrm{v}(-)$ group tended to be higher, but not significantly, both at $53.6 \%$, than $26.5 \%$ and $10.6 \%$, respectively, in the $\mathrm{v}(+)$ group.

Neural invasion (pn): Thirty-six cancers were analyzed, excluding 1 of unknown histopathology. The 3 - and 5-year survival rates in the pn(-) group were significantly higher, both at $71.4 \%$, than $17.9 \%$ on the 3 -year survival rate in the $\mathrm{pn}(+)$ group.

Survival rates by the presence or absence of histological invasion of other organs (extension to the surrounding tissue)

Direct intrahepatic invasion (pHinf): Thirty-three cancers were analyzed, excluding 4 of unknown histopathology. The 3- and 5-year survival rates in the pHinf(-) group were significantly higher, at $43.6 \%$ and $34.9 \%$, respectively, than $16.7 \%$ on the 3 -year survival rate in the $\mathrm{pHinf}(+)$ group.

Gallbladder-side invasion (pGinf): Thirty-six cancers were analyzed, excluding 1 of unknown histopathology. The 3- and 5-year survival rates in the pGinf $(-)$

A

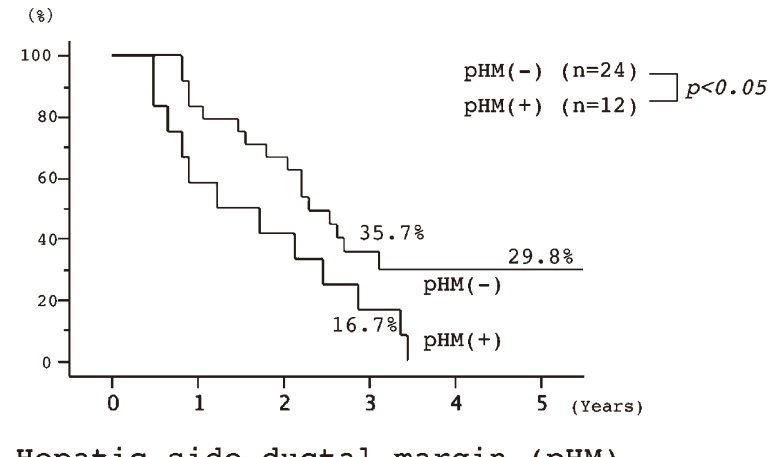

B

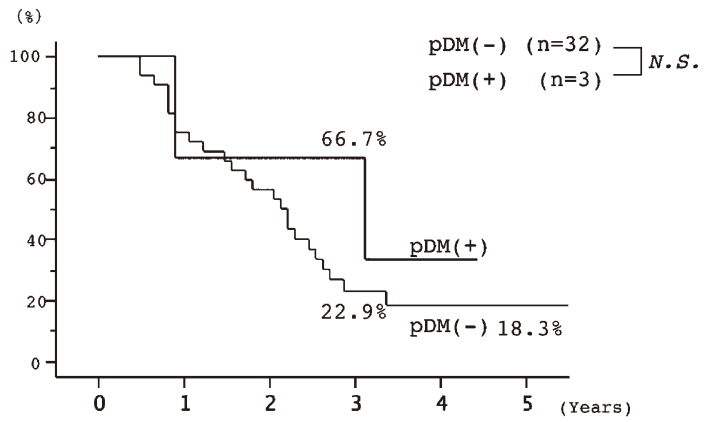

Duodenal-side ductal margin (pDM)

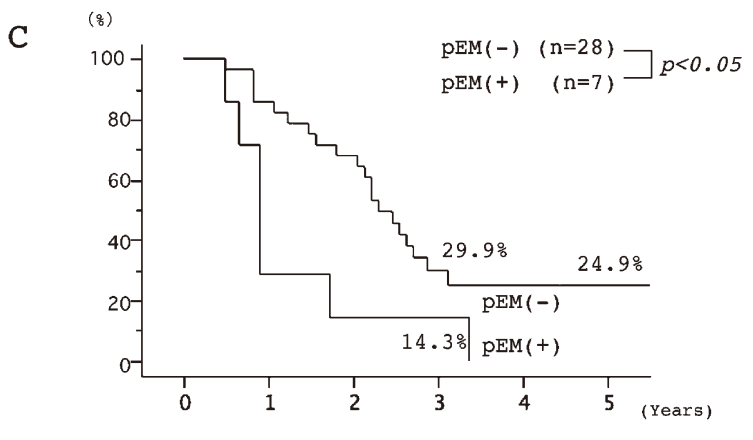

Excision plane margin (pEM)

Fig. 3. Survival rates by parameters related to the resection margin. 
group tended to be higher, but not significantly, at $34.7 \%$ and $27.8 \%$, respectively, than $18.2 \%$ and $0 \%$, respectively, in the $\operatorname{pGinf}(+)$ group.

Survival rates by the presence or absence of lymph node metastasis

Thirty-five cancers were analyzed, excluding 2 of unknown histopathology. The 3- and 5-year survival rates in the $\mathrm{pN}(-)$ group tended to be higher, but not significantly, at $35.4 \%$ and $28.3 \%$, respectively, than $21.4 \%$ and $7.1 \%$, respectively, in the $\mathrm{pN}(+)$ group.

Survival rates by the presence or absence of histological residual cancer in the resection margin

Hepatic-side ductal margin (pHM): Thirty-six cancers were analyzed, excluding 1 of unknown histopathology. The 3- and 5-year survival rates in the $\mathrm{pHM}(-)$ group were significantly higher, at $35.7 \%$ and $29.8 \%$, respectively, than $16.7 \%$ and $0 \%$, respectively, in the
$\mathrm{pHM}(+)$ group (Fig. 3A).

Duodenal-side ductal margin (pDM): Thirty-five cancers were analyzed, excluding 2 of unknown histopathology. The 3- and 5-year survival rates in the $\mathrm{pDM}(-)$ group were $22.9 \%$ and $18.3 \%$, respectively, and the 3 -year survival rate in the $\mathrm{pDM}(+)$ group was $66.7 \%$. No difference was noted in the survival rate (Fig. 3B).

Excision plane margin (pEM): Thirty-five cancers were analyzed, excluding 2 of unknown histopathology. The 3- and 5-year survival rates in the $\mathrm{pEM}(-)$ group were significantly higher, at $29.9 \%$ and $24.9 \%$, respectively, than $14.3 \%$ and $0 \%$, respectively, in the $\mathrm{pEM}(+)$ group (Fig. 3C).

\section{Survival rates by final stage}

Significant differences were noted among cumulative survival rates by final stage; in particular, the difference was marked between the stage I+II group and

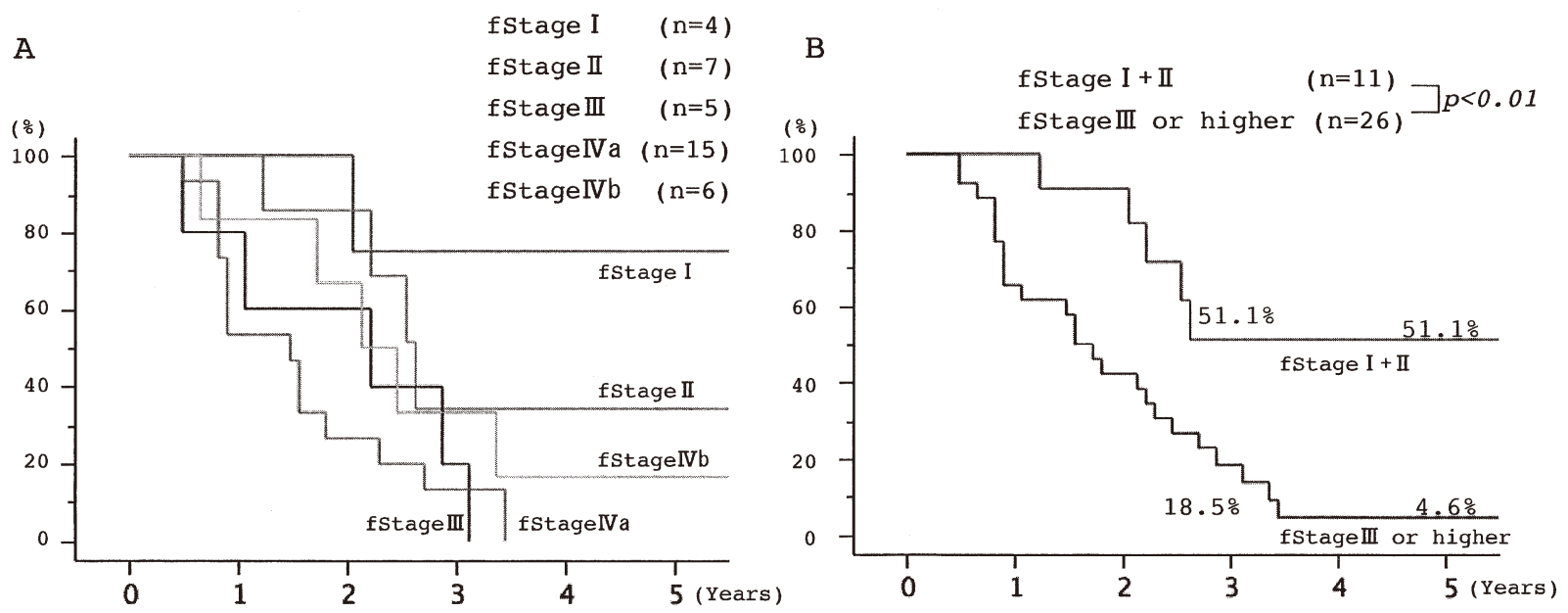

Fig. 4. Survival rates by final stage.

A

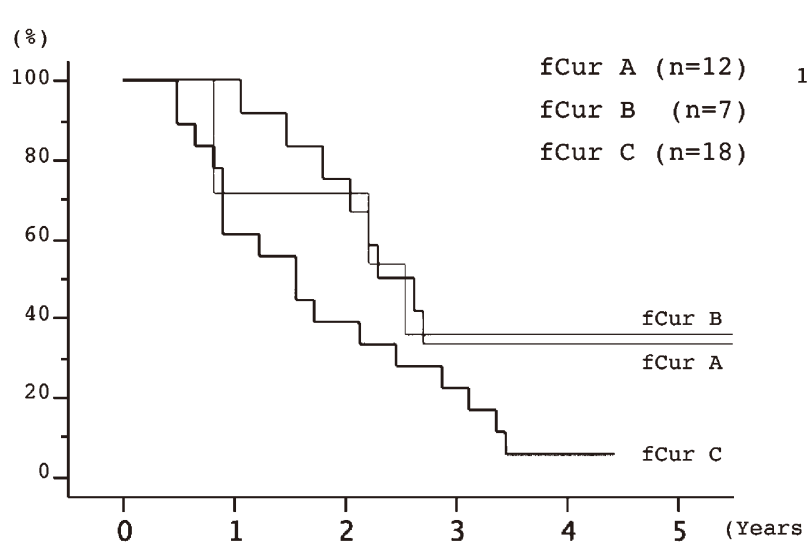

\section{B}

(응

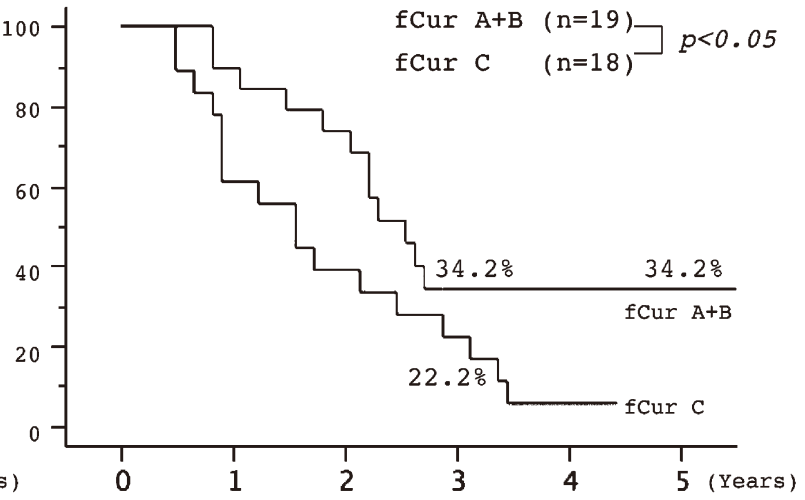

Fig. 5. Survival rates by final curability. 
the stage III or higher group (Fig. 4). The 3- and 5-year survival rates were both $51.5 \%$ in the final stage I/II group, and $18.5 \%$ and $4.6 \%$, respectively, in the final stage III or higher group.

\section{Survival rates by final curability}

The final curability A/B group had a significantly better prognosis than the final curability $\mathrm{C}$ group (Fig. 5). The 3- and 5-year survival rates were both $34.2 \%$ in the final curability A/B group, and the 3-year survival rate was $22.2 \%$ in the final curability $\mathrm{C}$ group.

\section{DISCUSSION}

\section{History of surgical treatment of hilar cholangiocarci- noma}

Hilar cholangiocarcinoma has attracted attention under the name of Klatskin tumor [2], since Klatskin [3] reported in 1965 that cancers in this location frequently caused fatal hepatic failure despite their small size and slow growth with infrequent metastasis. Since hilar cholangiocarcinomas are in an anatomically complex location, radical surgery is difficult to perform despite their small size, and they have been generally regarded as tumors that are anatomically difficult to treat [4]. Later, the anatomy of the hepatic hilum was gradually clarified [5-7], and aggressive resection including hepatectomy came to be performed [8-11]. The history of surgical treatment began in 1954 with Brown's success in the two-stage resection of the extrahepatic bile duct [12]. However, the so-called Longmire's operation [13] (involving internal fistulation by left intrahepatic cholangiojejunostomy) and the report of Terblanche et al. [14] that bypass surgery alone without local resection achieved long-term survival may have discouraged surgeons against trying surgical resection in the 1970s. The surgical technique of Cameron et al. [15] involving local bile duct resection followed by cholangiojejunostomy and percutaneous silicon tube stenting promoted resection therapy to some extent. Hart and White [16] developed a surgical technique in which a good visual field was ensured for adequate resection and reconstruction of the bile duct by resecting the anterior liver parenchyma at the hepatic hilum, the so-called inferior portion (S4a) of the medial segment of the left liver lobe. This was a step forward to approach the difficult-to-reach hilar bile duct. However, as was clarified by later studies of the local anatomy of the hepatic hilum, their resection technique inevitably left cancer behind in the resection margin. On the other hand, an attempt at radical tumor resection by hepatic lobectomy began in 1962 with left hepatic lobectomy by Altemeier [17]. In 1964, Quattlebaum first tried right hepatic lobectomy [18], in which Bird et al. first succeeded in 1969 [19]. In the latter 1960s, Haynes et al. [20] and Cady and Fortner [21] reported successful left hepatic lobectomy. In the 1970s, cases of successful left hepatic lobectomy were reported in succession. In 1973, Longmire et al. [22] succeeded in combined right hepatic trisegmentectomy and portal vein resection with end-to-end anastomosis reconstruction. In 1976, Fortner et al. [23] succeeded in left hepatic lobectomy followed by heterotopic liver transplantation, and the patient survived for 8 months. From 1980 onwards, many reports were published from Japan as well as from Europe and the United States, and the number of cases of combined portal vein and liver resection increased yearly, as reported by Tsuzuki et al. [24], Sakaguchi et al. [25], and Nimura et al. [26]. Caudate lobectomy is another important surgical factor in performing radical surgery for hilar cholangiocarcinoma. Blumgart et al. [27] first described concomitant caudate lobectomy in 1979, but did not clearly state the rationale for it. Later, Japanese researchers, Tsuzuki et al. [24], Mizumoto et al. [28], Iwasaki et al. [29], and Nimura et al. [30] successively published clinical study reports, and studies have demonstrated in more detail the clinically oriented local anatomy of the hepatic caudate lobe and the surgical anatomy of intrahepatic bile duct branches and the hepatic hilum [31-37], achieving a worldwide consensus about the rationality of caudate lobectomy in surgery for hilar cholangiocarcinoma $[38,39]$. In our department, we have performed concomitant caudate lobectomy in only $15(40.5 \%)$ of the 37 patients to date. However, Mizumoto et al. [40] pointed out the importance of caudate lobectomy for hilar cholangiocarcinoma in 1984, and further histopathological studies demonstrated the invasion of caudate lobe parenchyma and bile duct branches by hilar cholangiocarcinoma. Therefore, we consider that caudate lobectomy is essential in any surgical technique of hepatectomy performed for hilar cholangiocarcinoma, that is, left hepatic lobectomy, extended left hepatic lobectomy, right hepatic lobectomy, extended right hepatic lobectomy, right hepatic trisegmentectomy, or S4a+S5 liver segmentectomy [41]. Currently, we have a policy to perform concomitant caudate lobectomy whenever possible.

\section{Preoperative management}

Jaundice-reducing procedure: In addition to preop- 
erative management for general open abdominal surgery, meticulous care is needed particularly to avoid postoperative liver failure in patients with hilar cholangiocarcinoma. Most patients with hilar cholangiocarcinoma present with obstructive jaundice, and US and CT can demonstrate dilatation of some or all intrahepatic bile ducts. In Japan, it is taken for granted that jaundice reduction by percutaneous transhepatic biliary drainage (PTBD) [42] decreases postoperative complications $[8,43]$. With poor management of jaundice, the operative mortality is reported to be more than 20\% [44]. In principle, all segments of the liver except the caudate lobe should be drained by PTBD to perform selective cholangiography necessary for the diagnosis of the degree of cancer extension (described below) and to prevent segmental cholangitis. In performing PTBD, it is essential to puncture a site away from the lesion. PTBD of a bile duct close to the site of biliary stenosis, where a cancer may be present, increases the likelihood of cancer extension or dissemination along the fistula. What is worse, the bile duct near the PTBD catheter insertion site is intensely inflamed, and Glisson's capsules undergo scarring in clumps, making it difficult to image-diagnose the extent of cancer extension. In addition, these changes can be misidentified as cancer during surgery, and the separation from arteries and the portal vein becomes extremely difficult, thereby constituting a serious impediment to performing radical surgery. Therefore, PTBD in the vicinity of the hepatic hilum is virtually contraindicated, and a site well away from the stenosis should be punctured [45].

Management of liver failure: Postoperative liver failure is the most serious postoperative complication of hilar cholangiocarcinoma, and accounts for the majority of postoperative in-hospital deaths [46]. Unlike the normal liver, the obstructive-jaundiced liver has some residual dysfunction even if biliary drainage has been performed for jaundice reduction. Therefore, liver dysfunction may develop after major hepatectomy. Since there is no effective treatment for postoperative liver failure, preoperative prevention is important. In addition to adequate biliary drainage for jaundice reduction and cholangitis prevention, portal vein branch embolization has recently come to be performed aggressively [47]. The portal vein branch of the segment to be resected is embolized to promote the atrophy of that region and the compensatory hypertrophy of the predicted residual liver, and major hepatectomy is scheduled for a few weeks later [48-50]. The catheter is inserted by puncture for ultrasound-guided percutaneous transhepatic portal embolization (PTPE) [51,52] or by direct puncture of the ileocolic vein through a small laparotomy. A fibrin glue, ethanol, variceal sclerosing agent, Gelfoam, and embolizing coil are used as embolizing material. It is necessary to prevent the regurgitation of embolizing material, to embolize the site a little away from the predicted residual liver (as an inflammatory reaction may occur and make portal vein separation difficult), and to consider re-embolization if early re-canalization occurs in the embolized portal vein branch[53].

\section{Diagnosis}

To determine the surgical technique, it is important to determine the extent of cancer invasion. Nimura et al. analyzed the results of cholangiography, CT, and percutaneous transhepatic cholangioscopy (PTCS) in detail, diagnosed cancer invasion of caudate lobe bile-duct branches preoperatively, determined the liver segment to be resected, and performed combined hepatic segmentectomy and caudate lobectomy, thereby improving the curable resection rate and treatment results. Among various examinations, direct cholangiography by PTBD remains the current gold standard for the diagnosis of the degree of extension in the long (horizontal) axis of the bile duct [54], which is most important for surgical technique selection, and the fundamental diagnosis of the degree of extension is made by interpreting the narrowing, sclerosis, and irregularity of the bile duct wall. In this regard, sufficient care should be taken not to aggravate the general condition by segmental cholangitis due to excessive imaging [55]. Our department performs PTBD as the fundamental preoperative jaundice-reducing procedure, diagnosis the degree of extension of hilar cholangiocarcinoma mainly by direct cholangiography, clarifies the anatomical characteristics of the vessels in individual patients by 3D-CT, and performs PTPE according to the hepatic reserve, thereby trying to more safely perform extended resection to increase the radical curability of hilar cholangiocarcinoma.

\section{Resected patients}

The mode of local extension of hilar cholangiocarcinoma is generally described as that in the vertical and horizontal directions [56-59]. The degree of extension strongly influences the degree of cancer progression, that is, the prognosis. Regarding vertical extension, early biliary cancers, which are not exposed on the serosal surface, with an invasion depth of $m$ or $\mathrm{fm}$, and those with an invasion depth of ss have a better prognosis than those with a greater depth of invasion. However, as evident from the anatomical charac- 
teristics of the hilar bile ducts, cancers that have extended outside the wall of bile ducts tend to directly invade the anatomically adjacent liver parenchyma and vascular wall. In this regard, the histopathological parameters pHinf, $\mathrm{pPV}$, and $\mathrm{pA}$ come into question, strongly influence the possibility of curable resection, and become major factors leading to pEM-positivity in resected patients [56]. Cancer extension in the horizontal direction, that is, in the upper and lower directions along the long axis of bile ducts, is also frequently observed, and comes into question in determining the extent of surgical resection. The mode of extension in the horizontal direction is divided into extension in the stroma $(\mathrm{ly}, \mathrm{v}, \mathrm{pn})$ and that in the bile duct wall. The superficial extension type [60], which extends by replacing epithelium without duct wall thickening, and is difficult to recognize by imaging modalities, as well as a skip lesion [61], is rarely observed. Tumor cells are sometimes observed to extend in the stroma beyond the invasive front of the main tumor. These patterns of horizontal extension contribute to making it difficult to determine the extent of resection based on a negative resection margin (pHM, pDM). Some studies have reported that PTCS [62] and intraductal ultrasound (IDUS) [61] are useful to diagnose the degree of extension of the superficial extension type and skip lesion, while other studies have noted that the placement of a PTBD catheter makes it difficult to determine the boundary of the lesion because of bile-duct wall thickening and mucosal denuding [63-65], requiring precautions.

In this study, the group negative for both parameters (pHM, pEM) related to the resection margin had a significantly better prognosis. The node metastasisnegative group tended to have a better prognosis, with no significant difference in the survival rate, suggesting that even if lymph node metastasis is observed before or during surgery, effective (residual tumor-free) lymph node dissection would lead to curability, promising a good prognosis. Since no effective adjuvant therapy for cholangiocarcinoma has been established to date, these results indicate the critical importance of performing surgery achieving final curability A or B. Unfortunately, multivariate analysis yielded no significant results regarding which histopathological parameters most strongly influenced the cumulative survival rate. We are considering further studies in more patients to aid in the determination of a treatment policy for hilar cholangiocarcinoma.

In conclusion, patients with an early, histologically well-differentiated hilar cholangiocarcinoma who have undergone surgery achieving final curability A or
B can be expected to have a good long-term prognosis. Therefore, it is important not only to aim at early detection, but also to determine the extent of resection and surgical technique based on the accurate evaluation of cancer extension.

ACKNOWLEDGMENTS: We thank Prof. S. Aoyagi for his thoughtful review of this manuscript. We are also grateful to Prof. T. Kinoshita for his constant guidance and manuscript review, and to Prof. Emeritus T. Nakayama for his helpful advice. We are indebted to members of our laboratory for their cooperation in this work.

\section{REFERENCES}

1. Japanese Society of Biliary Surgery. General Rules for Surgical and Pathological Studies on Cancer of the Biliary Tract, 5th edn, Kanehara, Tokyo, pp 1-65, 2003. (in Japanese)

2. Mizumoto R. Treatment strategy for hilar carcinoma of the bile duct. Geka Chiryo 1999; 80:1255-1256. (in Japanese)

3. Klatskin G. Adenocarcinoma of hepatic duct at the bifurcation within the porta hepatis. An usual tumor; distinctive clinical and pathological features. Am J Med 1965; 38:241256.

4. Nimura Y. Treatment of hilar cholangiocarcinoma; present and future perspective. Geka 2003; 65:663-668. (in Japanese)

5. Couinaud C. Surgical anatomy of the liver revisited. selfpublication, Paris, pp 39-53, 1989.

6. Taoka D, and Kawarada Y. Surgical anatomy of the hepatic hilar area. J Jpn Surg Soc 2000; 101:386-392. (in Japanese)

7. Ishiyama S, Yamada S, Narushima Y, Yamaki T, Kunii Y et al. Surgical anatomy of the hilar bile duct. J Bil Panc 1999; 20:811-820. (in Japanese)

8. Nimura Y, Kamiya S, Kondo S, Nagino M, Uesaka K et al. Aggressive preoperative management and extended surgery for hilar cholangiocarcinoma; Nagoya experience. J Hepatobiliary Pancreat Surg 2000; 7:155-162.

9. Nakeeb A, Pitt HA, Sohn TA, Coleman J, Abrams RA et al. Cholangiocarcinoma. A spectrum of intrahepatic, perihilar, and distal tumors. Ann Surg 1996; 224:463-475.

10. Miyazaki M, Ito H, Nakagawa K, Ambiru S, Shimizu H et al. Aggressive surgical approaches to hilar cholangiocarcinoma; hepatic or local resection? Surgery 1998; 123:131136.

11. Burke EC, Jarnagin WR, Hochwald SN, Pisters PW, Fong $\mathrm{Y}$ et al. Hilar cholangiocarcinoma; patterns of spread, the importance of hepatic resection for curative operaton, and a presurgical clinical staging system. Ann Surg 1998; 228:385-394.

12. Brown G. Surgical removal of tumors of the hepatic duct. Postgrad Med 1954; 16:79-85.

13. Longmire WP Jr, and Lippman HN. Intrahepatic cholangiojejunostomy-an operation for biliary obstruction. Surg Clin N Amer 1956; 36:849-863.

14. Terblanche J, Saunders SJ, and Louw JH. Prolonged palliation in carcinoma of the main hepatic duct junction. Surgery 1972; 71:720-731. 
15. Cameron JL, Broe P, and Zuidema GD. Proximal bile duct tumors; surgical management with silastic transhepatic biliary stents. Ann Surg 1982; 196:412-419.

16. Hart MJ, and White TT. Central hepatic resection and anastomosis for stricture or carcinoma at the hepatic bifurcation. Ann Surg 1980; 192:299-304.

17. Mistilis S, and Schiff L. A case of jaundice due to unilateral hepatic duct obstruction with relief after hepatic lobectomy. Gut 1963; 4:13-15.

18. Quattlebaum JK, and Quattlebaum JK Jr. Malignant obstruction of the major hepatic ducts. Ann Surg 1965; 161:876-899.

19. Bird AD, Kerr GD, and Wynne-Jones G. Hepatic lobectomy for carcinoma of the hepatic duct. NZ Med J 1971; 73:351-354

20. Haynes CD, Gingrich GW, and Thorougham JC. Carcinoma of the bile duct; diagnosis and treatment. Am Surg 1964; 30:578-582.

21. Cady B, and Fortner JG. Surgical resection of intrehepatic bile duct cancer. Am J Surg 1969; 118:104-107.

22. Longmire WP, McArthur MS, Bastounis EA, and Hiatt J. Carcinoma of the extrahepatic biliary tract. Ann Surg 1973; 178:333-343.

23. Fortner JG, Kinne DW, Kim DK, Castro EB, Shiu MH et al. Vascular problems in upper abdominal cancer surgery. Arch Surg 1978; 109:148-153.

24. Tsuzuki T, Ogata Y, Iida S, Nakanishi I, Takenaka Y et al Carcinoma of the bifurcation of the hepatic ducts. Arch Surg 1983; 118:1147-1151.

25. Sakaguchi S, and Nakamura S. Surgery of the portal vein in resection of cancer of the hepatic hilus. Surgery 1986; 90:344-349.

26. Nimura Y, Hayakawa N, Kamiya J, Maeda S, Kondo S et al. Combined portal vein and liver resection for carcinoma of the biliary tract. Br J Surg 1991; 78:727-731.

27. Blumgart LH, Drury JK, and Wood CB. Hepatic resection for trauma, tumor and biliary obstruction. Br J Surg 1979; 66:762-769.

28. Mizumoto R, Kawarada Y, and Suzuki H. Surgical treatment of hilar carcinoma of the bile duct. Surg Gynecol Obstet 1986; 162:153-158.

29. Iwasaki Y, Okamura T, Ozaki A, Todoroki T, Takase Y et al. Surgical treatment for carcinoma at the confluence of the major hepatic ducts. Surg Gynecol Obstet 1986; $162: 457-464$

30. Nimura Y, Hayakawa N, Kamiya J, Kondo S, and Shionoya S. Hepatic segmentectomy with caudate lobe resection for bile duct carcinoma of the hepatic hilus. World J Surg $1990 ; 14: 535-544$

31. Suzuki H. Correlation and abnormalities of the blood vessels in Glisson's capsule: From the standpoint of hepatobiliary surgery. Nihon geka hokan 1982; 51:713-731. (in Japanese)

32. Kumon M. Problems in surgical treatment of hilar cholangiocarcinoma in relation to cancer extension into the caudate lobe biliary tree. Kanzo 1985; 26:1193-1199. (in Japanese)

33. Nimura Y, Hayakawa N, Hasegawa Y, Kamiya J, Kondo S et al. Problems in surgical treatment of hilar cholangiocarcinoma as judged from the degree of extension to caudate lobe bile-duct branches. J Jpn Surg Soc 1986; 87:10941097. (in Japanese)

34. Mizumoto R, and Suzuki H. Surgical anatomy of the hepatic hilum with special reference to the caudate lobe. World J Surg 1988; 12:2-10.

35. Kamiya J, Nimura Y, Hayakawa N, Maeda S, and Kondo S. Preoperative cholangiography of the caudate lobe; surgical anatomy and staging for biliary carcinoma. J Hepatobiliary Pancreat Surg 1994; 1:385-389.

36. Couinaud C. The paracaval segments of the liver. J Hepatobiliary Pancreat Surg 1994; 1:145-151.

37. Nimura Y. Surgical regional anatomy of the caudate lobe: Segments I and IX. J Bil Panc 1996; 17:1189-1196. (in Japanese)

38. Nimura Y, Hayakawa N, Kamiya J, Maeda S, Kondo S et al. Hilar cholangiocarcinoma; surgical anatomy and curative resection. J Hepatobiliary Pancreat Surg 1995; 2:239248

39. Su CH, Tsay SH, Wu CC, Shyr YM, King KL et al. Factors influencing postoperative morbidity, mortality, and survival after resection for hilar cholangiocarcinoma. Ann Surg 1996; 223:384-394.

40. Mizumoto R, Ogura Y, and Akasaka Y. Feature article: Surgery of bile duct cancer: Current status and problems of resection results. J Jpn Surg Assoc 1984; 39:1383-1388. (in Japanese)

41. Imayama H, Kinoshita H, Okuda K, Hara M, Hasuta K et al. Surgical treatment for hilar bile duct carcinoma. Geka Chiryo 1997; 76:845-848. (in Japanese)

42. Nakayama T, Ikeda A, and Okuda K. Percutaneous transhepatic drainage of the biliary tract. Technique and results in 104 cases. Gastroenterology 1977; 74:708-714.

43. Nagino M, Nimura Y, and Kamiya J. Preoperative management of hilar cholangiocarcinoma. J Hepatobiliary Pancreat Surg 1995; 2:215-223.

44. Sato T, Andoh H, Shibata S, Yasui O, Yoshioka M et al. Current standard treatments for hilar bile duct carcinoma. Geka 2001; 63:1565-1571. (in Japanese)

45. Nimura Y, Kamiya J, Kondo S, Nagino M, and Kanai M Technique of inserting multiple biliary drains and management. Hepatogastroenterology 1995; 42:323-331.

46. Nagino M, Kamiya J, Uesaka K, Sano T, Yamamoto H et al. Complications of hepatectomy for hilar cholangiocarcinoma. World J Surg 2001; 25:1277-1283.

47. Makuuchi M, Thai BL, Takayasu K, Takayama T, Kosuge $\mathrm{T}$ et al. Preoperative portal embolization to increase safety of major hepatectomy for hilar bile duct carcinoma: a preliminary report. Surgery 1990; 107:521-527.

48. Nagino M, Nimura Y, Kamiya J, Kondo S, Uesaka K et al. Changes in hepatic lobe volume in biliary tract cancer patients after right portal vein embolization. Hepatology $1995 ; 21: 434-439$

49. Uesaka K, Nimura Y, and Nagino M. Changes in hepatic lobar function after right portal vein embolization; an appraisal by biliary indocyanine green excretion. Ann Surg 1996; 223:77-83

50. Nagino M, Kamiya J, Kanai M, Uesaka K, Sano T et al. Right trisegment portal vein embolization for biliary tract carcinoma: technique and clinical utility. Surgery 2000; $127: 155-160$ 
51. Nagino M, Nimura Y, and Hayakawa N. Percutaneous transhepatic portal embolization using newly devised catheters: preliminary report. World J Surg 1993; 17:520-524.

52. Nagino M, Nimura Y, Kamiya J, Kondo S, and Kanai M. Selective percutaneous transhepatic embolization of the portal vein in preparation for extensive liver resection: the ipsilateral approach. Radiology 1993; 200:559-563.

53. Goto Y, Nagino M, and Nimura Y. Doppler estimation of portal blood flow after percutaneous transhepatic portal vein embolization. Ann Surg 1998; 228:209-213.

54. Shimizu H, Ito H, Kimura F, Togawa A, Ohtsuka M et al. Cholangiographic Diagnosis of Cancer Extension for Hilar Cholangiocarcinoma. Syoukakigazo 2004; 6:373-379. (in Japanese)

55. Kamiya J, and Nimura Y. Selective cholangiography (2) Hilar cholangiocarcinoma. Syoukakigazo 1999; 1:435-439. (in Japanese)

56. Nagai H, Kuroda K, Wada Y,Morioka Y, Noro $T$ et al. Pathology of Bile Duct Carcinoma at the Hepatic Hilus-Modes of Spread. J Bil Panc 1984; 5:1469-1481. (in Japanese)

57. Nagakawa $T$, Kitagawa $H$, Kayahara $M$, Ohta $T$, and Konishi I. Spreading patterns of hilar bile duct cancer. J Jpn Surg Soc 2000; 101:399-403. (in Japanese)

58. Ebata T, Watanabe H, Ajioka Y, Nishikura K, Date K et al. Extrahepatic Bile Duct Cancer: Correlation between macroscopic appearance and mode of spread. Syoukakigazo 1999; 1:165-172. (in Japanese)
59. Nagakawa T, and Ohta T. The mode of spread of extrahepatic bile Duct carcinoma. J. Bil. Panc. 1990; 11:333-338. (in Japanese)

60. Ojima H, and Sakamoto M. Clinicopathological features of hilar cholangiocarcinoma. Syoukakigazo 2004; 6:345-351. (in Japanese)

61. Kai M, Chijiiwa K, Tsuchiya K, Ouchida J, Eto T et al. A case of hilar bile duct cancer with skip lesion diagnosed correctly by intraductal ultrasonography (IDUS). J J B A 2004; 18:531-539. (in Japanese)

62. Maguchi H, Takahashi K, Katanuma A, T hayashi, A Yoshida et al. Evalustion of tumor extent and selection of surgical treatment for bile duct carcinomas. Syoukakigazo 2003; 5:367-374. (in Japanese)

63. Tamada K, Tomiyama T, Ichiyama M, Ohashi A, Wada S et al. Influence of biliary drainage catheter on bile duct wall thickness as measured by intraductal ultrasonography. Gastrointest Endosc 1998; 47:28-32.

64. Tamada K, Kanai N, Wada S, Tomiyama T, Ohashi A et al. Utility and limitations of intraductal ultrasonography in distinguishing longitudinal cancer extension along the bile duct from inflammatory wall thickening. Abdom Imaging 2001; 26:623-631.

65. Kamiya J, Nishio H, Arai T, Oda T, Nagino M et al. Indication of percutaneous transhepatic cholangioscopy (PTCS) and cholangioscopic biopsy. J Bil Panc 2003; 24:433-437. (in Japanese) 
